\title{
A autorregulação, avaliação e promoção da aprendizagem por meio da prática de recuperação da memória
}

\author{
Letícia Horn Oliveira ${ }^{1}$, https://orcid.org/0000-0001-5446-8344 \\ Lilian Milnitsky Stein'1, https://orcid.org/0000-0002-3599-0660
}

\begin{abstract}
Resumo
Este artigo pretende discutir a avaliação como promotora dos processos de aprendizagem em sala de aula, para além do exame. Para isso, traz a discussão sobre "o efeito de teste" como uma metodologia a serviço da consolidação da memória nos processos de ensino e autorregulação do estudo pelos estudantes. Testar com frequência conteúdos aprendidos pode otimizar o ensino e modificar a forma como hoje são conduzidos os processos em sala de aula. O conhecimento de estratégias metacognitivas de aprendizagem leva a um maior empoderamento dos alunos na gestão do aprendizado, consolidando o papel do professor como um mediador do conhecimento.
\end{abstract}

Palavras-chave: Avaliação; aprendizagem; memória.

\section{Self-regulation, evaluation, and promotion of learning through the practice of memory recovery}

\begin{abstract}
This article intends to discuss evaluation as a promoter of the learning processes in the classroom, in addition to the exam. For this, it brings the discussion about "the test effect" as a methodology in the service of the consolidation of memory in the processes of teaching and self-regulation of the study by the students. Frequently testing learned content can optimize teaching and modify the way classroom processes are conducted today. The knowledge of metacognitive strategies of learning leads to a greater empowerment of students in the management of learning, consolidating the role of the teacher as a mediator of knowledge.
\end{abstract}

Keywords: Evaluation; learning; memory.

\section{La autorregulación, evaluación y promoción del aprendizaje por intermedio de la práctica de recuperación de la memoria}

\section{Resumen}

En este artículo se intenta discutir la evaluación como promotora de los procesos de aprendizaje en sala de clase, para más allá del examen. Para eso, se hace una discusión sobre "el efecto de test" como una metodología a servicio de la consolidación de la memoria en los procesos de enseñanza y autorregulación del estudio por los estudiantes. Testar con frecuencia contenidos aprendidos puede optimizar la enseñanza y modificar la forma como hoy son conducidos los procesos en sala de clase. El conocimiento de estrategias meta cognitivas de aprendizaje lleva a un mayor empoderamiento de los alumnos en la gestión del aprendiz, consolidando el papel del profesor como mediador del conocimiento.

Palabras clave: Evaluación; aprendizaje; memoria.

1 Pontifícia Universidade Católica do Rio Grande do Sul, Programa de Pós-Graduação em Psicologia - Porto Alegre, RS, Brasil. 


\section{Introdução}

Quando falamos em avaliação em sala de aula logo nos remetemos à realização de testes gerais e provas, que estão a serviço de avaliar os conhecimentos dos alunos. Há também uma confusão entre os termos examinar e avaliar. A maneira como, frequentemente, as avaliações ocorrem hoje estão mais a serviço de uma detecção quantitativa do que o aluno conseguiu memorizar, para aquele momento em que o instrumento de avaliação é aplicado, do que para uma promoção da aprendizagem. Um exemplo disso são os exames aplicados aos alunos ao fim de cada bimestre letivo, que buscam examinar o passado, onde o estudante é desafiado a responder aquilo que já aprendeu. O exame aqui tem uma função classificatória, onde o foco não está no processo e sim no desempenho final, como ocorre num concurso público, por exemplo (Luckesi, 2011). Esse tipo de avaliação acaba assumindo um papel de controle, na medida em que os erros e acertos é que vão atestar uma boa aprendizagem, onde serão reconhecidos os conhecimentos dos alunos sobre determinados conteúdos (Oliveira \& Santos, 2005).

A avaliação pode, muitas vezes, se tornar um fator de interferência na aprendizagem, levando os alunos a estudarem especificamente os conteúdos que serão cobrados em uma prova. Tal situação pode acabar prejudicando o aprofundamento e mesmo a retenção das informações na memória de longo prazo (Karpicke \& Roediger, 2007). Ainda, se os erros não são trabalhados na relação professor-aluno com um feedback (retorno) adequado sobre o que o aluno compreendeu, não é possível avaliar a real efetividade da aprendizagem (Buriasco, 2000). O feedback é fundamental para que o aluno e professor revejam seus métodos de ensino e aprendizagem (Butler \& Roediger, 2008).

Portanto, uma avaliação realizada em etapas estanques acaba por desconsiderar o caráter processual da apropriação dos conceitos na relação de aprendizagem (Leite \& Kager,2009; Oliveira \& Santos, 2005; Souza \& Macedo, 2012; Souza, 2012) e perde seu caráter principal que deveria ser apontar caminhos para melhorar ações pedagógicas (Luckesi, 2011).Sendo assim, os resultados de uma avaliação deveriam ser utilizados para rever as metodologias do ensino, visando, sobretudo, a gestão do conhecimento e o caráter processual da aprendizagem (Correia \& Freire, 2010; Leite \& Kager, 2009; Luckesi, 2011).

Sasaki, Oliveira, Barreto e Rocha (2014) realizaram um estudo com alunos do Ensino Fundamental, no qual constatou que os estudantes percebem a avaliação como um procedimento estressante e de sobrecarga emocional, e não como algo que pudesse integrar a aprendizagem. O que acaba se fazendo nas escolas é exame e não uma avaliação, pois tem um caráter classificatório que pode levar ao fracasso escolar. Essa concepção de avaliação parece estar enraizada desde a própria formação profissional que os educadores receberam sobre o ato de avaliar, enquanto, ainda no papel de alunos (Luckesi, 2011; Cavalcanti Neto \& Aquino, 2009).

Quando tratamos de olhar para a avaliação como um caminho para o aprendizado estamos também trazendo à reflexão o processo de aprender a aprender. Boruchovitch (2014) ao refletir sobre o conceito de aprendizagem autorregulada faz uma crítica ao modo como trabalhamos o ensino, mesmo aquele que envolve a formação de professores, por se trabalhar pouco técnicas voltadas ao aprender a aprender. Para a autora o aprender a aprender e o desenvolvimento metacognitivo deveriam ser aspectos fundamentais quando se trata da estruturação de projetos psicopedagógicos escolares.

A aprendizagem autorregulada envolve estratégias cognitivas e metacognitivas e também aspectos afetivos e motivacionais no processo do aprender a aprender. Se os profissionais da educação não recebem esse tipo de conhecimento em seus cursos de formação, tampouco poderão orientar seus alunos sobre estratégias de aprendizagem (Santos \& Boruchovitch, 2009). Cabe reforçar aqui que, quando se trata de falar de profissionais da educação, estamos tratando dos profissionais de ensino de diferentes graus escolares.

Ao discutirmos aqui o processo de autorregulação da aprendizagem queremos também empoderar o aprendiz com ferramentas que facilitem compreender os mecanismos psicológicos que estão por trás do aprendizado e que dão suporte à capacidade de aprender a aprender (Boruchovitch, 2007; Oliveira,Boruchovitch \& Santos, 2009). A Psicologia Cognitiva, por meio da teoria do processamento da informação, auxilia na compreensão das estratégias metacognitivas utilizadas no planejamento, monitoramento e regulação do processo de aprender. Quando o aluno consegue se apropriar de estratégias cognitivas, ele passa a ser responsável pelo próprio aprendizado e o professor, conhecedor das técnicas, se torna um mediador deste conhecimento (Boruchovitch, 2007; Dembo, 2001).

Este artigo pretende trazer, portanto, uma discussão sobre os processos de avaliação em sala de aula, em que a testagem possa ser um meio para a aquisição de conhecimento. Para isso trataremos aqui dos pressupostos sobre o "efeito de teste"(traduzido do inglês testing effect), partindo dos princípios de funcionamento da memória para falar de aprendizagem e metacognição.

Pretende-se aqui considerar a avaliação como um aspecto promotor da gestão do conhecimento dos alunos e não como uma mera testagem de conhecimentos aprendidos. O que se quer discutir, prioritariamente, é a avaliação considerada como um processo facilitador na aprendizagem, e não um fim em si mesma.

\section{Aprendizagem autorregulada, memória e o uso de testes como ferramenta pedagógica}

A partir de princípios científicos do estudo da memória foram descobertos caminhos para se promover a aprendizagem dos alunos em sala de aula e a autorregulação de seus estudos. Um desses caminhos é prática de recuperação ou recordação de informações da memória.

Quando falamos de aprendizagem estamos também falando de memória, pois ambas estão implicadas. Ao rea- 
lizarmos a aquisição de um novo conteúdo estamos trabaIhando eminentemente com a memória de trabalho, que processa uma quantidade limitada de informação. Sendo assim, se é apresentado muito material novo aos alunos, isso pode vir a sobrecarregá-los (Barak, 2012). A forma como acontece esse aprendizado irá determinar se a informação será armazenada na memória de curto prazo (poucas horas de duração) ou armazenada na memória de longo prazo (meses ou anos). Sendo assim, quando falamos de uma aprendizagem significativa estamos também a observar quais os fatores que levam a uma boa consolidação da memória (Baddley, 2011; Cowan, 2008).

A informação na memória de curto prazo tem o registro máximo de três horas, que é o período no qual ela será lembrada. Sendo assim, se uma informação recebida não é bem consolidada, para ser armazenada na memória de longo prazo, seu conteúdo pode vir a ser esquecido (Butler \& Roediger, 2008).

Joly, Rodrigues, Bueno e Almeida (2015) realizaram uma pesquisa com 126 estudantes universitários da área de ciências exatas sobre competências de estudo. Na pesquisa foi identificado que os estudantes autorregulam seus estudos utilizando mais estratégias voltadas a ações tais como anotações em aula, organização de horários, leituras adicionais e exercícios, e menos em relação à estratégias metacognitivas voltadas à aprender como aprender. Essas estratégias envolveriam o monitoramento dos resultados do estudo pelo próprio aluno, de forma a comparar quais processos funcionariam melhor (Dantas \& Rodrigues,2013).

Nos últimos anos vários estudos tem descrito a importância dos testes repetidos para a efetiva organização das informações na memória de longo prazo, evidenciando que esta estratégia leva a maior recuperação de informações no futuro (Karpicke \& Blunt, 2014; Larsen, Butler, \& Roediger, 2013; Lyle \& Crawford, 2011; Karpicke \& Blunt, 2011; Karpicke \& Roediger, 2008; Roediger \& Karpicke, 2006; Smith, Blunt, Whiffen \& Karpicke,2016). Esses estudos têm mostrado que a recuperação (recordação) de uma determinada informação da memória aumenta a aprendizagem a longo prazo dessa informação, um fenômeno muitas vezes chamado de "efeito de teste". Esse efeito remete à importância de se testar um conteúdo, repetidas vezes, para que ele possa ser eficientemente armazenado na memória, facilitando sua posterior recordação e aplicação (Butler \& Roediger, 2007; McDaniel \& Masson, 1985).

McDaniel e Masson (1985) foram os primeiros a estudar a importância dos testes repetidos durante o aprendizado, para a consolidação da memória. Identificaram que uma série de testes de memória influencia mais fortemente a retenção da memória de longo prazo do que os estudos repetidos (McDaniel \& Masson, 1985; McDaniel, 1991).

Roediger e Karpicke (2006) realizaram um estudo com estudantes universitários onde eles foram convidados a realizar uma atividade com leitura. Foram divididos em pequenos grupos e nesses foram distribuídos pequenos textos para leitura (onde os participantes teriam um tempo fixo para ler os textos). Alguns grupos foram orientados a estudar o texto uma vez apenas e na sequência realizaram três testes de recordação livre sobre o texto, onde eram convidados a anotar em uma folha tudo o que lembravam do material lido. Outros grupos releram o mesmo texto três vezes, mas não foram testados sobre o material lido. Após cindo minutos de intervalo todos os grupos realizaram um teste final de recordação livre sobre o que lembravam do texto. O que se identificou nessa pesquisa foi que, passados cinco minutos, os grupos que haviam lido o texto repetidas vezes, lembravam de maior quantidade de informações do que os grupos que haviam lido apenas uma vez o texto e sido testados.

Porém, a pesquisa não foi encerrada nessa fase. Os mesmos estudantes também foram testados dois dias depois e uma semana depois, e o resultado foi surpreendente para os pesquisadores. Os grupos que tinham lido apenas uma vez o texto, mas sido testados em sequência três vezes, recordavam muito mais informações do que os grupos que tinham apenas lido o texto repetidas vezes, sem ser testados imediatamente depois. Desse modo os pesquisadores identificaram que os testes apresentam efeito positivo a longo prazo e previnem o esquecimento de informações, armazenadas na memória de longo prazo. A investigação constatou, portanto, que o estudo repetido e teste repetido implicaram resultados diferentes no teste final. Sendo assim, uma série de testes é mais benéfico do que uma série de estudos para fins de retenção das informações a longo prazo. Isso reforça a afirmação de que a prática de recuperação é o caminho para a promoção da memória e aprendizagem (Roediger \& Karpicke, 2006).

Esse mesmo experimento já foi replicado em outros países (Butler \& Roediger, 2008; Karpicke \& Roediger, 2007; Lipowski, Pyc, Dunlosky, \& Rawson, 2014; Lyle \& Crawford, 2011; McDermott, Agarwal, D’Antonio, Roediger, \& McDaniel 2014; Pyc \& Rawson, 2010). Em uma das pesquisas (Butler \& Roediger, 2008) foi realizada uma comparação entre grupos de estudantes adultos, utilizando técnicas de estudo repetido de material, testes de múltiplas escolha e teste de respostas curtas. Ao final de um mês, os estudantes que realizaram testes com respostas curtas tiveram uma performance de recuperação de informações, significativamente superior, aos alunos que apenas leram o material. A recuperação de informações também foi superior aos alunos que foram submetidos ao teste de múltipla-escolha. Esse resultado pode ser explicado porque o teste de resposta curta exige maior esforço mental na recuperação de informação do que o teste de múltipla escolha (Fazio, Agarwal, Marsh, \& Roediger, 2010).

Eisenkraemer, Jaeger e Stein (2013) realizaram um estudo de revisão sistemática com artigos publicados internacionalmente, entre os anos de 2006 a 2012 (época de grande publicação de investigações acerca do efeito de teste). Os resultados das investigações realizadas demonstraram que os testes podem ser extremamente benéficos para a retenção de memórias a longo prazo.

Também com crianças, os resultados são surpreendentes. Em uma investigação realizada com crianças do ensino fundamental (primeiro e terceiro ano), Lipowsky e cols (2014) identificaram benefícios na utilização de teste 
de repostas curtas na recuperação de informações a longo prazo. Também Karpicke, Blunt e Smith (2016) em um estudo realizado com 88 crianças do ensino fundamental, com diferentes níveis de compreensão de leitura e velocidade de processamento, verificaram resultados positivos com a prática de testagem pós leitura.

$\mathrm{O}$ efeito de teste pode ser utilizado tanto com materiais simples quanto mais complexos (Karpicke \& Aue, 2015). Jaeger, Eisenkraemer e Stein (2014) realizaram uma investigação no Brasil com crianças da terceira série do ensino fundamental, utilizando um pequeno texto contendo 321 palavras. O texto foi lido por todos os 69 participantes e as crianças foram divididas em dois grupos: um grupo de teste e outro grupo de reestudo. As tarefas de memória foram realizadas durante o turno escolar, dentro da sala de aula e na presença dos colegas e professores. Num primeiro momento todos leram o texto duas vezes. Em seguida realizaram um teste de matemática simples (para distração) por 3 minutos. Na sequência o grupo de teste realizou um teste de recordação com pistas que consistia em uma frase do texto com uma lacuna em branco a ser preenchida com uma palavra. Já o grupo de reestudo teve a tarefa de reler o texto mais duas vezes. Sete dias depois ambos os grupos foram testados com um teste de múltipla escolha contendo quatro opções para marcar uma das alternativas como correta. As crianças que realizaram o teste inicial com recordação apresentaram maior retenção de conteúdo (87\%) do que as crianças que haviam somente reestudado o texto (53\%).

\section{Benefícios da prática de recuperação (recordação) no processo ensino-aprendizagem}

O efeito de teste pode ser aplicado tanto a ambientes educacionais como clínicos, sendo utilizado no reforço da aprendizagem nos alunos e redução dos déficits de memória em populações clínicas (Pastötter \& Baüml, 2014). Karpicke e Roediger (2008) evidenciaram o papel crítico da prática de recuperação (recordação) na consolidação da aprendizagem. A prática da recordação pode otimizar e muito o tempo que os alunos levam para assimilar os conteúdos, seja em sala de aula ou estudos individuais. As pesquisas de Karpicke e Roediger (2007) constataram que a prática de testagem leva a uma menor necessidade de repetição de estudos, como a leitura repetida de um texto, por exemplo, para a assimilação de um conteúdo. A prática de estudo repetido, que ocorre, por exemplo, quando lemos várias vezes um texto, para estudar para uma prova, é uma estratégia que não garante, necessariamente, o armazenamento das informações na memória de longo prazo.

Uma hipótese é que os testes melhoram a aprendizagem porque multiplicam o número de rotas de recordação para eventos armazenados, ou seja, funcionam como pistas para a recordação posterior da informação. Isso ocorre pelo esforço feito quando as memórias são recuperadas (McDaniel \& Fisher,1991; McDaniel \& Masson,1985; Pyc \& Rawson, 2010). De acordo com essa hipótese, quanto maior esforço estiver envolvido na recordação de uma memória, mais extensivo será o reprocessamento das informações memorizadas. Sendo assim, os testes que exigem maior dificuldade para recuperar a informação são os mais benéficos para retenção das informações a longo prazo (McDaniel, Roediger, \& McDermott, 2007).

A outra hipótese para a eficácia do efeito de teste baseia-se no processo de transferência da informação. Nesse sentido, o desempenho da memória é reforçado na medida em que os processos cognitivos necessários, durante a aprendizagem de um novo conteúdo, coincidem com os requeridos durante a recuperação. Os processos envolvidos na evocação ou resgate de informações da memória, partindo da realização de um teste inicial após uma leitura, por exemplo, aumentam a performance em um teste final em maior grau do que os processos envolvidos no estudo repetido do material (várias leituras, por exemplo). Haveria, portanto, uma compatibilidade entre as operações envolvidas durante a fase de teste e de aprendizagem (Roediger, 1990; Roediger \& Butler, 2011).

Agarwal, D’Antonio, Roediger, McDermott e McDaniel (2014) realizaram uma pesquisa com 1.408 alunos do ensino médio sobre a prática de recuperação (recordação de informações da memória utilizando o "efeito de teste"). Nesse estudo foi constatado que $92 \%$ dos estudantes tiveram benefícios com a prática de recuperação no sentido de facilitar a sua aprendizagem e $78 \%$ relataram ter ficado menos nervosos quando submetidos a exames. Nesse sentido, além do benefício prático para consolidação das informações na memória de longo prazo, a prática de testagem traz também um componente emocional, na medida em que deixa os alunos menos ansiosos. Percebe-se aí, portanto, o quanto seria benéfico a utilização dos testes frequentes de forma a monitorar e promover o aprendizado (Roediger \& Karpicke, 2006)

Mas não basta apenas testar, também aqui o feedback é fundamental. O feedback, aqui entendido como o retorno da compreensão do aluno sobre a informação ministrada é importante, inclusive, para se rever se a relação ensino-aprendizagem está adequada. Se é realizado apenas um teste final sobre todo o conteúdo aprendido em dois meses, no caso de um bimestre letivo, por exemplo, podem ficar lacunas pelo caminho (Agarwal et al., 2013). Testes frequentes em sala de aula com feedback são efetivos para a retenção e aprendizado, seja utilizando testes de respostas curtas, de recordação livre ou testes de múltipla escolha. Os testes frequentes ainda incentivam os alunos a estudarem de forma contínua e não apenas para um exame final (McDermott \& cols., 2014; McDaniel \& cols., 2007).

Testar logo após ter estudado um conteúdo, protege contra interferências de um conteúdo que será aprendido na sequência, pois o teste ajuda a consolidar a informação na memória. A prática de recuperação da informação por meio de um teste de curta duração, por exemplo, melhora a organização das informações (Pastötter \& Baüml, 2014).

Roediger, Putnam e Smith (2011) listaram alguns benefícios da prática de testagem na recuperação (recordação) da informação: a recuperação facilita a retenção 
posterior; o teste identifica lacunas no conhecimento; testar produz melhor organização do conhecimento; testar melhora a transferência de conhecimentos para novos contextos; testes melhoram a monitoração metacognitiva; testes impedem interferências de material anterior quando se aprende um novo material; testes fornecem feedback para instrutores; testes frequentes incentivam os alunos a estudar.

Esses benefícios independem de grau de ensino, podendo ser aplicados ao ensino fundamental, médio e superior. A prática de recuperação por meio de testagem é uma estratégia de aprendizagem que se concentra em obter informações da mente e trazê-la à consciência. Não necessita de recurso tecnológico, dinheiro ou maior tempo da sala de aula.

Quando pensamos em ensino-aprendizagem tipicamente nos focamos em introduzir informações à mente dos estudantes, com a esperança que elas sejam aderidas. Nesse propósito os professores fornecem desde leituras, apresentam vídeos, incentivam o registro de anotações por meio de revisões ou resumos. Essas estratégias não são ruins, porém, acabam levando em conta muitas vezes, apenas a aprendizagem de curto prazo (Agarwal \& cols.,2013; Butler \& Roediger, 2008).

Outra questão a salientar é que o fato de uma informação vir facilmente à mente, parecendo estar fluente, não garante que ela não seja esquecida (Agarwal \& cols.,2013). Por exemplo, se estudamos com afinco para uma prova específica, as chances de sermos bem sucedidos na avaliação é grande. Porém, dependendo do método escolhido para estudo, não iremos garantir que esta memória esteja com seu armazenamento garantido a longo prazo.

A prática de recordação, por meio de testagens frequentes logo após o estudo, torna a prendizagem esforçada e desafiadora, pois requer maior esforço mental. Por exemplo, ter que recordar livremente uma resposta a uma questão sobre ciência, melhora a aprendizagem em maior medida do que procurar a resposta para a mesma questão em um livro. Resumindo, quanto mais difícil a prática de recuperação, melhor para a aprendizagem de longo prazo (Agarwal \& cols., 2013).

Carpenter, Pashler e Cepeda (2009) convidaram alunos do ensino médio a estudar um texto sobre a história dos Estados Unidos. Os fatos históricos foram estudados e testados por alguns estudantes com intervalos de uma e dezesseis semanas. Paralelo a isso, outros estudantes somente reestudaram o texto nesses mesmos períodos. Após nove meses de intervalo, todos os alunos receberam um teste sobre os fatos estudados. Os estudantes que haviam lido o texto e na sequência testados, apresentaram uma meIhor aprendizagem sobre o conteúdo do que os alunos que apenas tinham lido o texto repetidas vezes.

Além disso, Wissman, Rawson e Pyc (2011) constataram, em uma pesquisa realizada com estudantes universitários, que testar conteúdos interfere positivamente na aquisição de novos conteúdos. Nesse estudo eles solicitaram aos estudantes de dois grupos que estudassem três sessões de texto com conteúdos diferentes em cada ses- são, mas o mesmo texto para ambos os grupos. Os textos tinham relação de conteúdo entre si. Um grupo (grupo-teste) foi testado com perguntas de recordação livre nas duas sessões (1 e 2), imediatamente após a leitura dos textos e o outro grupo foi orientado apenas a ler os textos das sessões 1 e 2 (os mesmos textos do grupo-teste). Quando os grupos foram submetidos a uma terceira sessão (3) com um texto diferente,mas igual para os dois grupos, ambos tiveram que responder a perguntas de recordação livre (respostas curtas). Porém, o que foi constatado é que o grupo que foi testado nas duas primeiras sessões recuperou mais informações na terceira sessão do que o grupo que não havia sido testado. Uma hipótese para este resultado é que os testes melhoram a consciência sobre o próprio aprendizado (consciência metacognitiva) estimulando por si só a adoção de estratégias mais eficazes de codificação da informação (Pyc \& Rawson,2010).

Os testes utilizados na prática de recordação podem ser desde respostas curtas, problemas práticos, questões de múltipla escolha e testes com partes do texto e lacunas em branco a ser preenchidas (teste de recordação com pistas). $\mathrm{O}$ mais importante aqui é o ato de buscar informações de nossas mentes e trazê-las à consciência (Agarwal \& cols., 2013).

A recuperação é um processo ativo e dinâmico de aprendizagem e ao mesmo tempo simples, tal como solicitar que os alunos recordem e escrevam, em uma linha, informações sobre o que se lembram da aula anterior (Pastötter \& Baüml, 2014). Sendo assim, não é o teste especificamente que promove a aprendizagem, mas sim os processos que ocorrem durante a testagem. O que promove a aprendizagem é o ato de recuperar informações da memória (Smith \& cols, 2016).

Para a efetividade do processo de recuperação através de testagem, no entanto, se recomenda que se faça um feedback. Pesquisa de McDermott e cols. (2014), com alunos de ensino médio nos Estados Unidos, resultou na confirmação de que tanto o teste de curta duração, como de múltipla escolha são efetivos como estratégia de recuperação da informação para respostas em exames posteriores.

Sendo o efeito de teste um meio para melhorar a aprendizagem e retenção de longo prazo, sugere-se que os alunos sejam testados (ou auto-testados) repetidamente enquanto aprendem. Além do benefício do retorno sobre a aprendizagem para um estudo futuro, a recuperação da informação beneficia fortemente a retenção. Em contextos educacionais o "efeito de teste", pode ser utilizado com crianças, jovens e adultos, beneficiando estudantes de baixa e alta capacidade e sendo aplicada a uma ampla variedade de situações (Pastötter,Weber, \& Bäuml, 2013).

Portanto, os métodos de ensino que estimulem a recuperação da informação, previamente armazenada na memória, podem ser muito úteis para a retenção de sucesso a longo prazo e, consequentemente, para a aprendizagem. Ao mesmo tempo, é uma prática que não envolve ocupar mais tempo da sala de aula, mas trata, eminentemente, de tornar o momento de ensino em sala de aula mais efetivo (Agarwal \& cols., 2013). 
Durante a livre recuperação de informações da memória por exemplo, não é necessária a utilização de um teste estruturado. Os estudantes aqui tem a tarefa de buscar da memória, livremente, tudo o que lembram do conteúdo estudado e isso, inclusive, pode trazer melhores resultados para o aprendizado do que testes de respostas curtas. É uma tarefa que não exige treinamento ou prepração prévia dos professores e nem dos alunos (Smith \& cols., 2016).

Smith e cols (2016) identificaram que fornecer testes com pistas favorece a prática de recordação livre posterior.Por exemplo,realizar um teste contendo uma frase sobre o texto estudado,com lacuna em branco para preencher e depois, em seguida, uma prática de recordação livre, pode ser ainda mais eficaz para o aprendizado, facilitando significativamente a retenção de conteúdo na memória de longo prazo. Isso ocorre porque aos alunos ampliam sua estrutura organizacional.

Em uma investigação realizada por Liu e Reder (2016) com estudos de neuroimagem do cérebro foi possível identificar os benefícios da prática de recuperação. Os pesquisadores identificaram uma maior atividade cerebral quando exercida a prática de recuperação em relação à atividade de reestudo, tanto nos hemisférios esquerdo e direito do cérebro. Sendo assim, foi possível identificar os benefícios fisiológicos da prática de recuperação, devido ao esforço que a prática exige.

A recuperação de informações, por meio de testagens frequentes, colabora com a autorregulação da aprendizagem dos alunos com efeitos duradouros a longo prazo. Destaca-se também a importância da prática de recuperação da memória para a transferência da aprendizagem para outros contextos, seja no ensino escolar ou treinamento profissional (Butler,2010).

\section{Considerações finais}

O objetivo principal deste artigo foi discutir uma nova maneira de olhar para a temática da avaliação, como sendo um instrumento que faça parte do processo de aprendizagem e não como um fim. Rever o estigma de que avaliação e exame são sinônimos foi a primeira proposta. Para esse fim sugerimos como um caminho a ser seguido a utilização de testes frequentes em sala de aula, de forma a facilitar o monitoramento metacognitivo dos estudantes.

A prática de recuperação da informação é uma ferramenta de aprendizagem simples e ao mesmo tempo poderosa e que está a serviço dos educadores tanto para uso em sala de aula quanto para autorregulação do estudo dos estudantes. Nesse sentido, os testes aqui cumprem uma função educativa, sem um caráter classificatório como nos exames bimestrais ou finais de curso. Seu papel aqui é o de promover a aprendizagem, facilitando a retenção de informações na memória de longa duração. O estudo repetido, forma usual de estudo adotada por grande parte dos estudantes, além de tomar mais tempo dos alunos, não garante que a informação será aprendida da forma mais eficaz e duradoura.
A prática de recuperação será mais efetiva se houver o retorno (feedback) sobre a compreensão do aluno, seja para rever um erro ou consolidar o acerto. Recuperar com frequência uma informação aprendida, por meio da testagem, facilita e muito o armazenamento e recordação posterior desse conteúdo. $O$ processo de ensinar em pequenos passos, por meio da utilização de testes frequentes, guiando a prática do estudante, se mostra um caminho favorável para empoderar o aluno no seu processo de aprendizagem de forma mais independente e autônoma.

Apesar dos inúmeros benefícios da testagem como prática de autorregulação dos estudos há carência de pesquisas e estudos com escalas que possam avaliar as estratégias de aprendizagem utilizadas pelos estudantes, bem como pelos professores (Oliveira \& cols., 2009). O que se sabe é que a utilização de estratégias metacognitivas está diretamente relacionada à um bom desempenho acadêmico, independentemente do grau escolar.

Portanto, proporcionar aos estudantes caminhos para a autonomia nos processos de aprendizagem é um caminho a ser seguido quando se fala em uma educação contemporânea e no uso de metodologias ativas. Isso se faz necessário para que o professor possa, cada vez mais, ocupar um papel de mediador no ensino-aprendizagem e o aluno se torne autor ativo de sua formação. Sugere-se, assim, investigações que possam avaliar como os estudantes, de diferentes níveis de ensino (regular e profissionalizante), podem se beneficiar da utilização da prática de autorregulação da aprendizagem, por meio da prática de recuperação da memória. Novas pesquisas, com uma amostra significativa da população, podem trazer subsídios para o desenvolvimento de políticas educacionais mais efetivas. É importante, também, considerar diferentes formas de testar a aprendizagem que sejam, ao mesmo tempo, atrativas e desafiadoras, levando os alunos a um maior engajamento e motivação para aprender.

\section{Referências}

Agarwal, P.K., Roediger, H.L; McDaniel, M.A.; McDermott, K.B (2013). How to use retrieval practice to improve learning. St.Louis: Washington University.

Agarwal, P.K.; D’Antonio, L.; Roediger III, H.L.; McDermott, K.B.; McDanieL, M.A (2014). Classroom-based programs of retrieval practice reduce middle school and high school students' test anxiety. Journal of Applied Research in Memory and Cognition, 3, 131-139

Barak, R. (2012) Principles of Instruction: Research-Based Strategies That All Teachers Should Know. American Educator, 36 (1), 12-19, 39

Baddley, A. (2011). Memória. Porto Alegre: Artmed.

Boruchovitch, E. (2007). Aprender a Aprender: propostas de intervenção em estratégias de aprendizagem. Educação Temática Digital, 8(2), 156-167 
Boruchovitch, E. (2014). Autorregulação da aprendizagem: contribuições da psicologia educacional para a formação de professores. Psicologia Escolar e Educacional, 18(3), 401409 doi:10.1590/2175-3539/2014/0183759

Buriasco, R.L.C. (2000). Algumas considerações sobre avaliação educacional. Avaliação Educacional, 22, 155-178.

Butler, A.C. (2010). Repeated Testing Produces Superior Transfer of Learning Relative to Repeated Studying. Journal of Experimental Psychology: American Psychological Association Learning, Memory, and Cognition, 36 (5), 1118-1133 doi: 10.1037/a0019902

Butler, A.C.; Roediger, H.L (2008). Feedback enhances the positive effects and reduces the negative effects of multiple-choice testing. Memory \& Cognition, 36 (3), 604-616 doi: 10.3758/MC.36.3.604

Carpenter, S.K.; Pashler, H.; Cepeda, N.J. (2009). Using tests to enhance 8th grade students' retention of U. S. history facts. Applied Cognitive Psychology, 23, 760-771

Cavalcanti Neto, A.L.G.; Aquino, J.L.F. (2009). A avaliação da aprendizagem como um ato amoroso: o que o professor pratica? Educação em Revista, 25(2), 223-240.

Correia, M.S.M.; Freire, A.M.M.S. (2010). Práticas de avaliação de professores de ciências físico-químicas do ensino básico. Ciência \& Educação, 16(1), 1-15.

Cowan, N. (2008).What is the diferences between long-term, shortterm, and working memory? Progress in Brain Research, 169, 323338.

Dantas, C.; Rodrigues, C.C. (2013). Estratégias metacognitivas como intervenção psicopedagógica para o desenvolvimento do automonitoramento. Revista Psicopedagogia, 30(93), 2026-2035

Dembo, M.H. (2001). Learning to teach is not enough: future teachers also need to learn to learn. Teacher Education Quaterly, 28(4) 23-35.

Eisenkraemer, R.E.; Jaeger, A.; Stein, L.M. (2013). A systematic review of the testing effect in learning. Paidéia, 23(56), 397-406. doi:10.1590/1982-43272356201314

Fazio,L.K.; Agarwal, P.K.; Marsh, E.J.; Roediger, H.L. (2010). Memorial consequences of multiple-choice testing on immediate and delayed tests. Memory \& Cognition, 38(4), 407-418. doi:10.3758/ MC.38.4.407

Jaeger, A., Eisenkraemer, R.E., Stein, L.M. (2014):Test-enhanced learning in third-grade children, Educational Psychology: An International Journal of Experimental Educational Psychology. do i: $10.1080 / 01443410.2014 .963030$

Joly, M.C.R.A; Rodrigues, C.F.F; Bueno, J.M.P; Almeida, L.S. (2015). Competência de estudo para uma amostra universitária da área de exatas.Psicologia Escolar e Educacional, 19(1), 23-29.
Karpicke, J.D.; Aue, W.R. (2015). The Testing Effect is Alive and Well with Complex Materials. Educational Psychology Review, 27, 317326 doi: 10.1007/s10648-015-9309-3

Karpicke, J.D.; Blunt, J.R. (2011). Retrieval practice produces more learning than elaborative studying with concept mapping. Science, 331, 772-775. doi: 10.1126/science.1199327

Karpicke, J.D.; Blunt, J.R. (2014). Learning With Retrieval-Based Concept Mapping. Journal of Educational Psychology, 106 (3), 849-858 doi: 10.1037/a0035934

Karpicke, J.D.; Blunt, J.R.; Smith, M.A. (2016) Retrieval-Based Learning: Positive Effects of Retrieval Practice in Elementary School Children. Frontiers in Psychology, 7,350.

Karpicke, J. D.; Roediger, H. L.III. (2007). Repeated retrieval during learning is the key to long-term retention. Journal of Memory and Language, 57(2), 151-162. doi:10.1016/j.jml.2006.09.004

Karpicke, J.D.; Roediger, H.L.III. (2008).The critical importance of retrieval for learning. Science, 319, 966-968. doi: 10.1126/ science. 1152408

Larsen, D.P.; Butler, A.C.; Roediger, H.L.(2013). Comparative effects of test-enhanced learning and self-explanation on long-term retention. Medical Education, 47, 674-682. doi: 10.1111/medu.12141

Leite, S.A.S.; Kager, S. (2009). Efeitos aversivos das práticas de avaliação da aprendizagem escolar. Ensaio: Avaliação e Políticas Públicas em Educação, 17(62), 109-34. doi:10.1590/S010440362009000100006

Liu, X.L.; Reder, L.M. (2016). fMRI exploration of pedagogical benefits of repeated testing: when more is not always better. Brain and Behavior, 6(7), doi: 10.1002/brb3.476

Lipowski, S.T.; Pyc, M.A.; Dunlosky, J.; Rawson, K.A. (2014). Establishing and Explaining the Testing Effect in Free Recall for Young Children. Developmental Psychology, 50 (4), 994-1000

Lyle, K.B.; Crawford, N.A. (2011). Retrieving essential material at the end of lectures improves performance on statistics exams. Teaching of Psychology, 38, 94-97. doi: 10.1177/0098628311401587

Luckesi. C.C. (2011). Avaliação da aprendizagem: componente do ato pedagógico. São Paulo: Cortez

McDaniel, M.A.; Fisher, R.P. (1991). Tests and test feedback as learning sources. Contemporary Educational Psychology, 16, 192201

McDaniel, M.A.; Masson, M.E.J. (1985). Altering memory representations through retrieval. Journal of Experimental Psychology: Learning, Memory and Cognition, 11, 371-85. doi: 10.1037/0278- 7393.11.2.371 
McDaniel, M.A.; Roediger, H.L.III; McDermott, K.B. (2007). Generalizing test-enhanced learning from the laboratory to the classroom. Psychonomic Bulletin \& Review, 14(2), 200-206. doi:10.3758/BF03194052

McDermott, K.B.; Agarwal, P.K.; D’Antonio, L.; Roediger,L.H.; McDaniel, M.A. (2014). Both Multiple-Choice and Short-Answer Quizzes Enhance Later Exam Performance in Middle and High School Classes. Journal of Experimental Psychology: Applied, 20 (1), 3-21

Oliveira, K.L.; Boruchovitch, E.; Santos, A.A.A. (2009). Estratégias de aprendizagem e desempenho acadêmico: evidências de validade. Psicologia: Teoria e Pesquisa, 25(4), 531-536.

Oliveira, K.L.; Santos, A.A.A. (2005). Avaliação da aprendizagem na Universidade. Psicologia Escolar e Educacional, 9(1), 37-46

Pastötter,B.; Baüml, K-Z.,T. (2014). Retrieval practice enhances new learning: the forward effect of testing. Frontiers in Psychology, 5, 1-5 doi:10.3389/fpsyg.2014.00286

Pastötter, B.; Weber, J.; Bäuml, K.H.T. (2013). Using testing to improve learning after severe traumatic brain injury. Neuropsychology, 27, 280-285. doi: 10.1037/a0031797

Pyc, M.A.; Rawson, K.A. (2010). Why testing improves memory: mediator effectiveness hypothesis. Science, 330-335. doi: 10.1126/ science. 1191465

Roediger H. L.; Butler, A.C. (2011). The critical role of retrieval practice in long-term retention. Trends Cogn Sci, 15, 20-27

Roediger, H.L.II; Karpicke, J.D. (2006). Testenhanced learning: Taking memory tests improves longterm retention. Psychological Science, 17(3), 249-255. doi:10.1111/j.1467-9280.2006.01693.x
Roediger, H.L.III; Putnam, A.L.; Smith, M.A. (2011).Ten benefits of testing and their applications to educational practice. Psychology of Learning and Motivation, 55, 1-36. doi 10.1016/B978-0-12387691-1.00001-6

Roediger, H L.III. (1990). Implicit memory: Retention without remembering. American Psychologist, 45(9),1043-1056. doi:10.1037/0003-066X.45.9.1043

Santos, O.J.X.; Boruchovitch, E. (2009). Estratégias de aprendizagem na formação de professores: uma análise da produção científica. Educação PUC-RS, 32 (3), 346-354

Sasaki, K.; Oliveira, L.C.P.; Barreto, M.O.; Rocha, N.M.F. (2014). Percepções de estudantes do ensino fundamental sobre sua avaliação de aprendizagem. Psicologia Escolar e Educacional, 18(1), 77-86.

Smith, M.A.; Blunt, J.R.; Whiffen, J.W.; Karpicke, J.D. (2016) Does Providing Prompts During Retrieval Practice Improve Learning? Applied Cognitive Psychology, 30(4), 544-553. doi: 10.1002/acp.3227.

Souza, A.M.L. (2012). Avaliação da aprendizagem no ensino superior: aspectos históricos. Exitus, 2(1),231-254

Souza, A.M.L; Macedo, M.C.S. (2012). Avaliação da aprendizagem e inclusão escolar: a singularidade a serviço da coletividade. Psicologia Escolar e Educacional, 16(2), 283-290.

Wissman, K.T.; Rawson, K.A.; Pyc, M.A. (2011). The interim test effect: Testing prior material can facilitate the learning of new material. Psychonomic Bulletin \& Review, 18, 1140-1147.

Recebido em: 08/09/2016

Aprovado em: 10/04/2017

\section{Sobre as autoras}

Letícia Horn Oliveira (leticiaholiveira@yahoo.com.br)

Doutoranda em Psicologia. Pontifícia Universidade Católica do Rio Grande do Sul (PUCRS). Porto Alegre- RS. https://orcid.org/0000-00015446-8344

Lilian Milnitsky Stein (lilian@pucrs.br)

Professora titular no programa de Pós Graduação em Psicologia.Pontifícia Universidade Católica do Rio Grande do Sul (PUCRS). Porto AlegreRS. https://orcid.org/0000-0002-3599-0660 